\title{
Decomposing dense bipartite graphs into 4-cycles
}

\author{
Nicholas J. Cavenagh \\ Department of Mathematics \\ The University of Waikato \\ Private Bag 3105 \\ Hamilton 3240, New Zealand \\ nickc@waikato.ac.nz
}

Submitted: Jun 8, 2013; Accepted: Feb 11, 2015; Published: Feb 25, 2015

Mathematics Subject Classification: 05C51, 05D99

\begin{abstract}
Let $G$ be an even bipartite graph with partite sets $X$ and $Y$ such that $|Y|$ is even and the minimum degree of a vertex in $Y$ is at least $95|X| / 96$. Suppose furthermore that the number of edges in $G$ is divisible by 4 . Then $G$ decomposes into 4-cycles.
\end{abstract}

\section{Introduction}

The order and size of a graph $G$ indicate the number of vertices and edges in $G$, respectively. All graphs are assumed to be simple (i.e. no loops or multiple edges) unless otherwise stated. Given a graph $G$, a set $\mathcal{D}$ of subgraphs of $G$ is said to be a decomposition of $G$ if each edge of $G$ is an edge of precisely one element of $\mathcal{D}$. If, furthermore, each element of $\mathcal{D}$ is isomorphic to a fixed graph $H$, we say that $G$ decomposes into $H$.

We discuss related results along two strands: the problem of finding decompositions of large graphs with high minimum degree and the problem of decomposing bipartite graphs into 4-cycles (and related decompositions).

\subsection{Dense decompositions}

In its most general form, the decomposition problem is NP-complete. That is, deciding whether an arbitrary graph $G$ decomposes into a given graph $H$ is NP-complete (unless $H$ has at most 2 edges; [7]). However, what if we allow the order and size of $G$ to be arbitrarily large (relative to $H)$ ? There are two necessary conditions for a decomposition that may fail no matter how large $G$ is relative to $H$ :

(C1) If $G$ decomposes into $H$ then the size of $H$ divides the size of $G$.

(C2) If $G$ decomposes into $H$ and $d$ is the greatest common divisor of the degrees of vertices in $H$, then $d$ must divide the degree of each vertex in $G$. 
On the other hand, if $G$ and $H$ satify conditions (C1) and (C2) above we say that $G$ is $H$-divisible.

Wilson [12] showed that these necessary conditions are sufficient for sufficiently large complete multigraphs:

Theorem 1. (Theorem 24.10 of [4]) For a fixed graph $H$ and integer $\lambda$, there exists an integer $N(H, \lambda)$ such that if $n>N(H, \lambda)$ and $\lambda K_{n}$ is $H$-divisible, then $\lambda K_{n}$ decomposes into $H$.

In this note we are interested in the problem of showing that graphs which are both sufficiently large and sufficiently dense (specifically, sufficiently large minimum degree) are decomposable. Indeed, Wilson's result above has been generalized to the dense case first by Gustavsson [8] and more recently in a submitted work [2]:

Theorem 2. $[8,2]$ Given a non-empty graph $H$, there exists a positive constant $\gamma$ ( a function of $H)$ such that if a graph $G$ has $n$ vertices, minimum degree $\left(1-\gamma+o_{n}(1)\right) n$ and $G$ is $H$-divisible, then $G$ decomposes into $H$.

This powerful theorem has been used to develop a host of related results and generalizations. These include showing decompositions $\mathcal{D}$ of sufficiently dense and large graphs where:

- $\mathcal{D}$ is a set of closed trails of arbitrary lengths [1];

- Each graph in $\mathcal{D}$ is isomorphic to a graph from a fixed (and finite) list [5].

The constant $\gamma$ in Theorem 2 is small; even if $H$ is a 3-cycle, the constant $\gamma$ is $10^{-24}$ [8], (improved to 0.044 in [2]). In general, if $H$ has $k$ vertices then $\gamma$ is $10^{-37} k^{-94}$ ([8], improved to $\left(9 k^{10}\right)^{-1}$ in [2]). We may compare this to a conjecture by Nash-Williams [10] that any graph $G$ of order $n$ and minimum degree at least $3 n / 4$ decomposes into 3 -cycles (so long as $G$ is 3-cycle-divisible.)

Asymptotically optimal lower bounds for the minimum degree of a $H$-divisible graph $G$ to ensure the existence of a $H$-decomposition have been obtained in the case when $H$ has a vertex of degree 1 and $H$ is bipartite [13]; and $H$ is an even cycle of length at least 6 [2]. In both of these cases the minimum degree bound is $n / 2\left(1+o_{n}(1)\right)$. (Indeed it is not hard to show this can't be improved if $H$ is connected.)

Such a bound cannot be obtained for 4-cycles; one can construct arbitrarily large graphs of minimum degree $3 n / 5-1$ which satisfy (C1) and (C2), but do not decompose into 4-cycles (see [13]; attributed to Winkler and Kahn). In [3] it is shown that if $G$ is 4-cycle-divisible and has minimum degree $31 n / 32\left(1+o_{n}(1)\right)$ then $G$ decomposes into 4-cycles; the fraction $31 / 32$ is improved to $2 / 3$ in [2].

Finally, in [3] it is shown that if $G$ is a 4-cycle-divisible bipartite graph with partite sets $X$ and $Y$ (each of size $n$ ) and minimum degree $31 n / 32\left(1+o_{n}(1)\right)$, then $G$ decomposes into 4-cycles. The results in this paper, in contrast, allow the partite sets to have different sizes. 


\subsection{Decompositions of complete bipartite graphs into 4-cycles and related decompositions}

We next discuss what is known about related decompositions of complete (rather than sufficiently dense) graphical structures. If we take a broad net there are dozens of papers which are in some way related to decompositions of bipartite graphs into 4-cycles; rather than making a comprehensive survey, we mention only the few which are most directly related. Determining necessary and sufficient conditions for the decomposition of a complete bipartite graph $K_{m, n}$ into 4-cycles is easy. Applying necessary conditions (C1) and (C2), $m$ and $n$ must be even. These conditions suffice; simply partition the vertices from each partite set into pairs, any two such pairs from different partite sets induce a 4-cycle. The resultant 4-cycles form a decomposition.

The problem becomes more challenging if we specify a constraint that no pair of 4cycles shares more than one vertex; such decompositions are monogamous and necessary and sufficient conditions for them are given in [9]. These structures are of interest because of their relation to symmetric $H$-squares and self-orthogonal 1-factorizations [9].

Necessary and sufficient conditions to decompose $G$ into $H$ are known for the following pairs $G$ and $H$ :

- $G$ is a complete bipartite graph and $H$ is a cycle of fixed length ([11]);

- $G$ is a complete multipartite graph and $H$ is a 4-cycle $([6])$.

\section{The general approach}

The main result of the paper is the following.

Theorem 3. Let $G$ be a bipartite graph with partite sets $X$ and $Y$ such that $|X|=m$, and $|Y|=n$ (where $n$ is even and $m, n \geqslant 2$ ), each vertex of $G$ has even degree and the minimum degree of a vertex in $Y$ is at least $d m$, where $d=95 / 96$. Then $G$ decomposes into 4-cycles.

We give a proof of the above theorem in Section 4; in this section we outline our general approach.

It is convenient to present the details of our proof in terms of an array representation. To this end, suppose that a bipartite graph $G$ with partite sets $X=\left\{x_{1}, x_{2}, \ldots, x_{m}\right\}$ and $Y=\left\{y_{1}, y_{2}, \ldots, y_{n}\right\}$ has a decomposition $\mathcal{D}=\left\{H_{1}, H_{2}, \ldots, H_{\ell}\right\}$, for some $l \geqslant 1$. Then we may construct a corresponding array $M=M(\mathcal{D})$ of dimensions $m \times n$, where the cell in row $i$ and column $j$ contains the entry $k$ (if the edge $\left\{x_{i}, y_{j}\right\}$ belongs to the subgraph $H_{k}$ ) or 0 (if the edge $\left\{x_{i}, y_{j}\right\}$ does not belong to $G$ ). We say that $M(\mathcal{D})$ is the decomposition array corresponding to the decomposition $\mathcal{D}$.

Next, suppose that $\mathcal{D}_{1}$ and $\mathcal{D}_{2}$ are disjoint, non-empty decompositions of the same graph $G$ (for our purposes some subgraph of the bipartite graph we are trying to decompose). Then we refer to the pair $\left(\mathcal{D}_{1}, \mathcal{D}_{2}\right)$ as a trade and we say that $\mathcal{D}_{1}$ trades with $\mathcal{D}_{2}$. 
Our approach to prove Theorem 3 is as follows. Let $G$ be the bipartite graph we wish to decompose into 4 -cycles. We start by placing a 0 is each cell of $M$ which corresponds to an edge that does not belong to $G$. Next, partition the rows and columns of $M$ of into pairs $\mathcal{P}_{R}$ and $\mathcal{P}_{C}$ (possibly with one row omitted, if the number of rows is odd). The intersection of each $P_{1} \in \mathcal{P}_{R}$ and $P_{2} \in \mathcal{P}_{C}$ gives a $2 \times 2$ subarray; if none of these 4 cells contain 0 the corresponding edges form a 4-cycle in $G$. We let the set of such 4-cycles be $\mathcal{C}_{0}$. Any cells corresponding to edges from $\mathcal{C}_{0}$ are initially classified as available. All remaining cells are classified as unavailable.

Next, we pack as many 4-cycles as possible into the edges of $G$ not used by cycles in $\mathcal{C}_{0}$. Let $\mathcal{C}_{1}$ be the set of such 4 -cycles. Finally, the remaining edges of $G$ decompose into a set of even-length cycles (which we call $\mathcal{C}_{2}$ ).

The 4-cycles of $\mathcal{C}_{1}$ are never changed in our construction. However the 4-cycles in $\mathcal{C}_{0}$ are sometimes combined with cycles from $\mathcal{C}_{2}$ to form a trade with a set of 4 -cycles only. Each time we do this, any available cells used are reclassified as unavailable. The minimum degree condition will ensure there are always enough available cells to choose from to make the necessary trades.

The following properties (P1) and (P2) will be invariant to the application of our trades. For each column $c$ of $M$ and for each pair $P=\left\{r, r^{\prime}\right\} \in \mathcal{P}_{R}$, the two cells $(r, c)$ and $\left(r^{\prime}, c\right)$ are either both available or both unavailable (property $\left.(\mathrm{P} 1)\right)$. The total number of available cells within a pair of rows from $\mathcal{P}_{R}$ is always divisible by 4 (property $(\mathrm{P} 2))$. These properties ensure that after all trades are completed, within each pair of rows $P \in \mathcal{P}_{R}$ any remaining available cells easily decompose into 4-cycles.

\section{Some useful trades and a bit of graph theory}

In this section we build up the necessary tools and methods to prove our main result (Theorem 3). Many of the constructions are most easily understood by studying examples first; we encourage the reader to look ahead to the various examples given. The following lemma shows how to trade a cycle of length divisible by 4 and a specified configuration of 4-cycles with a set of 4-cycles only.

Lemma 4. Let $\left\{r_{1}, r_{2}, \ldots, r_{2 k}\right\}$ (respectively, $\left\{c_{1}, c_{2}, \ldots, c_{2 k}\right\}$ ) be a set of distinct rows (respectively, columns) of a decomposition array $M$, where $k \geqslant 2$. For each $i, 1 \leqslant i \leqslant$ $k-1$, let $\left\{r_{2 i-1}^{\prime}, r_{2 i}^{\prime}\right\}$ be a set of distinct rows of $M$. Let $T$ be a $4 k$-cycle on the set of cells

$$
\left\{\left(r_{1}, c_{1}\right),\left(r_{2 k}, c_{2 k}\right)\right\} \cup\left\{\left(r_{i}, c_{i+1}\right),\left(r_{i+1}, c_{i}\right) \mid 1 \leqslant i \leqslant 2 k-1\right\} .
$$

For each $i, 1 \leqslant i \leqslant k-1$, let $S_{i}$ be the set of cells

$$
S_{i}=\left\{\left(r_{2 i-1}^{\prime}, c_{j}\right),\left(r_{2 i}^{\prime}, c_{j}\right) \mid 2 i-1 \leqslant j \leqslant 2 i+2\right\}
$$

in effect two disjoint 4-cycles. Suppose furthermore that the sets of cells $T, S_{1}, S_{2}, \ldots, S_{k-1}$ are pairwise disjoint. Then the edges corresponding to the union of these sets of cells decompose into 4-cycles. 
Proof. For each $i$ such that $1 \leqslant i \leqslant k-1$, observe that the eight cells from the rows $r_{2 i}$, $r_{2 i+1}$ and $r_{2 i}^{\prime}$ may be used by two 4 -cycles. For each $i$ such that $1 \leqslant i \leqslant k-2$, the four cells in rows $r_{2 i-1}^{\prime}$ and $r_{2 i+1}^{\prime}$ and columns $c_{2 i+1}$ and $c_{2 i+2}$ may be used by a 4 -cycle. The eight remaining cells belong to the set

$$
\left\{\left(r_{1}, c_{1}\right),\left(r_{1}, c_{2}\right),\left(r_{1}^{\prime}, c_{1}\right),\left(r_{1}^{\prime}, c_{2}\right)\right\} \cup\left\{\left(r_{2 k}, c_{2 k-1}\right),\left(r_{2 k}, c_{2 k}\right),\left(r_{2 k-3}^{\prime}, c_{2 k-1}\right),\left(r_{2 k-3}^{\prime}, c_{2 k}\right)\right\}
$$

which give the remaining two 4-cycles in the decomposition.

Example 5. We illustrate the method of the above lemma with $k=3$ :

\begin{tabular}{|c|c|c|c|c|c|c|}
\hline & $c_{1}$ & $c_{2}$ & $c_{3}$ & $c_{4}$ & $c_{5}$ & $c_{6}$ \\
\hline$r_{1}$ & 1 & 1 & & & & \\
\hline$r_{2}$ & 2 & & 2 & & & \\
\hline$r_{3}$ & & 3 & & 3 & & \\
\hline$r_{4}$ & & & 4 & & 4 & \\
\hline$r_{5}$ & & & & 5 & & 5 \\
\hline$r_{6}$ & & & & & 6 & 6 \\
\hline$r_{1}^{\prime}$ & 1 & 1 & 7 & 7 & & \\
\hline$r_{2}^{\prime}$ & 2 & 3 & 2 & 3 & & \\
\hline$r_{3}^{\prime}$ & & & 7 & 7 & 6 & 6 \\
\hline$r_{4}^{\prime}$ & & & 4 & 5 & 4 & 5 \\
\hline
\end{tabular}

Corollary 6. Let $C$ be a cycle of length divisible by 4 (and at least 8) within a decomposition matrix $M$ (satisfying (P1) and (P2)), where the cells of $C$ are classified as unavailable. Suppose that for any subset of 4 columns from $M$, there exist 2 elements of $\mathcal{P}_{R}$ which are available in all of these columns. Then, there exists a trade $\left(\mathcal{D}_{1}, \mathcal{D}_{2}\right)$ where $\mathcal{D}_{1}$ consists of $C$ and some 4-cycles whose edges correspond to available cells with a set $\mathcal{D}_{2}$ of 4-cycles only. Moreover, after reclassifying any available cells from $\mathcal{D}_{1}$ as unavailable, the matrix $M$ still satisfies properties (P1) and (P2).

The following technical lemma makes it possible to assume that any two cycles with lengths congruent to $2(\bmod 4)$ share at most one vertex within a given partite set.

Lemma 7. Let $C_{1}$ and $C_{2}$ be two edge-disjoint cycles in a bipartite graph (with partite sets $X$ and $Y)$, each of length congruent to $2(\bmod 4)$. Suppose that $C_{1}$ and $C_{2}$ share at least two common vertices from partite set $Y$. Then $\left\{C_{1}, C_{2}\right\}$ may be traded with either $\left\{C_{3}, E\right\}$, where $C_{3}$ is a cycle of length divisible by 4 and $E$ is an even graph, or $\left\{C_{4}, C_{5}, E\right\}$, where $C_{4}$ and $C_{5}$ have length congruent to 2 (mod 4) and share at most one common vertex within $Y$.

Proof. Consider the even graph $G$ formed by combining the edges of $C_{1}$ and $C_{2}$. If $G$ contains a cycle of length divisible by 4 , we are done. Otherwise, since the number of edges in $G$ is divisible by 4 , there are at least two edge-disjoint cycles in $G$. Let $C$ and $C^{\prime}$ be a pair of such cycles such that the total number of edges in $C$ and $C^{\prime}$ is minimum. 
Suppose that $C$ and $C^{\prime}$ intersect at exactly two vertices ( $v$ and $w$, say). If $v$ and $w$ belong to distinct partite sets, the lemma is satsified. Otherwise there exist four paths within the union of these cycles, each starting at $v$ and ending at $w$ with an even number of edges, with internal vertices pairwise disjoint. Since $C$ and $C^{\prime}$ both have lengths congruent to $2(\bmod 4)$, at least two of these paths have length divisible by 4 . Thus we may join two paths to create a cycle of length of length divisible by 4 , and we are done.

Next, suppose that $C$ and $C^{\prime}$ intersect at at least three vertices. Thus there exist vertices $v, w$ and $x$ which belong to both $C$ and $C^{\prime}$ and paths $v P_{1} w$ and $w P_{2} x$ within $C$ such that no internal vertices of $P_{1}$ or $P_{2}$ belong to $C^{\prime}$. There thus exist paths $v Q_{1} w$, $w Q_{2} x$ and $x Q_{3} v$ within $C_{2}$ such that the set of paths $\left\{P_{1}, P_{2}, Q_{1}, Q_{2}, Q_{3}\right\}$ are pairwise internally vertex-disjoint.

However, the paths $P_{1}, P_{2}, Q_{1}$ and $Q_{2}$ together form a pair of edge-disjoint cycles, contradicting the minimality assumed in the first paragraph of this proof. Hence $C$ and $C^{\prime}$ share at most one vertex in the partite set $Y$, as required.

Corollary 8. Any even, bipartite graph with partite sets $X$ and $Y$ has a decomposition $\mathcal{D}$ into cycles such that if $C_{1}, C_{2} \in \mathcal{D}$ and $C_{1}$ and $C_{2}$ each have length congruent to 2 (mod 4), then $C_{1}$ and $C_{2}$ share at most one common vertex within the partite set $Y$.

The next lemma trades two cycles which are either column-disjoint or share at most one column (and have length at least 10$)$, each of length congruent to $2(\bmod 4)$ and a set of specified 4-cycles with a set of 4-cycles only.

Lemma 9. Let $k, l \geqslant 1$. Within a decomposition matrix $M$, let

$$
\left\{r_{1}, r_{2}, \ldots, r_{2 k+1}\right\},\left\{r_{1}^{\prime}, r_{2}^{\prime}, \ldots, r_{2 l+1}^{\prime}\right\},\left\{u_{1}, u_{2}, u_{3}, u_{4}\right\},\left\{s_{1}, s_{2}, \ldots, s_{2 k-2}\right\},\left\{s_{1}^{\prime}, s_{2}^{\prime}, \ldots, s_{2 l-2}^{\prime}\right\}
$$

be sets of distinct rows and let

$$
\left\{c_{1}, c_{2}, \ldots, c_{2 k+1}, c_{1}^{\prime}, c_{2}^{\prime}, \ldots, c_{2 l+1}^{\prime}\right\}
$$

be a set of distinct columns (with possibly $c_{2 l+1}^{\prime}=c_{2 k+1}$ if $k, l \geqslant 2$ ). Let $T$ and $T^{\prime}$ be disjoint sets of cells corresponding to cycles of length $4 k+2$ and $4 l+2$, respectively. Let $T$ occupy the set of cells

$$
\left\{\left(r_{1}, c_{1}\right),\left(r_{2 k+1}, c_{2 k+1}\right)\right\} \cup\left\{\left(r_{i}, c_{i+1}\right),\left(r_{i+1}, c_{i}\right) \mid 1 \leqslant i \leqslant 2 k\right\}
$$

and let $T^{\prime}$ occupy the set of cells

$$
\left\{\left(r_{1}^{\prime}, c_{1}^{\prime}\right),\left(r_{2 l+1}^{\prime}, c_{2 l+1}^{\prime}\right)\right\} \cup\left\{\left(r_{i}^{\prime}, c_{i+1}^{\prime}\right),\left(r_{i+1}^{\prime}, c_{i}^{\prime}\right) \mid 1 \leqslant i \leqslant 2 l\right\} .
$$

Let $U_{1}$ and $U_{2}$ be the sets of cells given by:

$$
\begin{aligned}
& U_{1}=\left\{\left(u_{i}, c_{j}\right),\left(u_{i}, c_{j}^{\prime}\right) \mid 1 \leqslant i \leqslant 2,1 \leqslant j \leqslant 3\right\}, \\
& U_{2}=\left\{\left(u_{i}, c_{j}\right),\left(u_{i}, c_{j}^{\prime}\right) \mid 3 \leqslant i \leqslant 4,2 \leqslant j \leqslant 3\right\} .
\end{aligned}
$$


For each $i, 1 \leqslant i \leqslant k-1$, let $S_{i}$ be the set of cells

$$
S_{i}=\left\{\left(s_{2 i-1}, c_{j}\right),\left(s_{2 i}, c_{j}\right) \mid 2 i \leqslant j \leqslant 2 i+3\right\} .
$$

For each $i, 1 \leqslant i \leqslant l-1$, let $S_{i}^{\prime}$ be the set of cells

$$
S_{i}^{\prime}=\left\{\left(s_{2 i-1}^{\prime}, c_{j}^{\prime}\right),\left(s_{2 i}^{\prime}, c_{j}^{\prime}\right) \mid 2 i \leqslant j \leqslant 2 i+3\right\} .
$$

Suppose furthermore that each of the above sets of cells are pairwise disjoint. Then the edges corresponding to the union of these sets of cells decompose into 4-cycles.

Proof. The following sets of cells each correspond to 4-cycles:

$$
\begin{array}{ll}
\left\{\left(r_{1}, c_{1}\right),\left(r_{1}, c_{2}\right),\left(u_{1}, c_{1}\right),\left(u_{1}, c_{2}\right)\right\}, & \left\{\left(r_{2}, c_{1}\right),\left(r_{2}, c_{3}\right),\left(u_{2}, c_{1}\right),\left(u_{2}, c_{3}\right)\right\}, \\
\left\{\left(r_{1}^{\prime}, c_{1}^{\prime}\right),\left(r_{1}^{\prime}, c_{2}^{\prime}\right),\left(u_{1}, c_{1}^{\prime}\right),\left(u_{1}, c_{2}^{\prime}\right)\right\}, & \left\{\left(r_{2}^{\prime}, c_{1}^{\prime}\right),\left(r_{2}^{\prime}, c_{3}^{\prime}\right),\left(u_{2}, c_{1}^{\prime}\right),\left(u_{2}, c_{3}^{\prime}\right)\right\}, \\
\left\{\left(u_{1}, c_{3}\right),\left(u_{1}, c_{3}^{\prime}\right),\left(u_{4}, c_{3}\right),\left(u_{4}, c_{3}^{\prime}\right)\right\}, & \left\{\left(u_{2}, c_{2}\right),\left(u_{2}, c_{2}^{\prime}\right),\left(u_{4}, c_{2}\right),\left(u_{4}, c_{2}^{\prime}\right)\right\} .
\end{array}
$$

The remaining 4-cycles in the decomposition may be found in a similar manner to Lemma

\begin{tabular}{|c|c|c|c|c|c|c|c|c|c|c|c|c|}
\hline & $c_{1}$ & $c_{2}$ & $c_{3}$ & $c_{4}$ & $c_{5}$ & $c_{1}^{\prime}$ & $c_{2}^{\prime}$ & $c_{3}^{\prime}$ & $c_{4}^{\prime}$ & $c_{5}^{\prime}$ & $c_{6}^{\prime}$ & $c_{7}^{\prime}$ \\
\hline$r_{1}=r_{1}^{\prime}$ & 1 & 1 & & & & 6 & 6 & & & & & \\
\hline$r_{2}=r_{2}^{\prime}$ & 2 & & 2 & & & 7 & & 7 & & & & \\
\hline$r_{3}=r_{3}^{\prime}$ & & 3 & & 3 & & & 8 & & 8 & & & \\
\hline$r_{4}=r_{4}^{\prime}$ & & & 4 & & 4 & & & 9 & & 9 & & \\
\hline$r_{5}=r_{5}^{\prime}$ & & & & 5 & 5 & & & & 10 & & 10 & \\
\hline & & & & & & & & & & 11 & & 11 \\
\hline$r_{7}^{\prime}$ & & & & & & & & & & & 12 & 12 \\
\hline$u_{1}$ & 1 & 1 & 13 & & & 6 & 6 & 13 & & & & \\
\hline$u_{2}$ & 2 & 14 & 2 & & & 7 & 14 & 7 & & & & \\
\hline$u_{3}$ & & 15 & 15 & & & & 16 & 16 & & & & \\
\hline$u_{4}$ & & 14 & 13 & & & & 14 & 13 & & & & \\
\hline$s_{1}=s_{1}^{\prime}$ & & 15 & 15 & 5 & 5 & & 16 & 16 & 17 & 17 & & \\
\hline$s_{2}=s_{2}^{\prime}$ & & 3 & 4 & 3 & 4 & & 8 & 9 & 8 & 9 & & \\
\hline & & & & & & & & & 17 & 17 & 12 & 12 \\
\hline$s_{4}^{\prime}$ & & & & & & & & & 10 & 11 & 10 & 11 \\
\hline
\end{tabular}
4 .

Example 10. We illustrate the previous lemma with an example. In our example to save space we have made many rows equal, however in general this is not a necessary condition in the construction.

In the next lemma we deal with the case of one 6-cycle and another cycle with length congruent to $2(\bmod 4)$, overlapping at one column.

Lemma 11. Let $l \geqslant 1$. Within a decomposition array $M$, let $\left\{r_{1}, r_{2}, r_{3}\right\},\left\{r_{1}^{\prime}, r_{2}^{\prime} \ldots, r_{2 l+1}^{\prime}\right\}$, $\left\{u_{1}, u_{2}, u_{3}, u_{4}\right\}$ and $\left\{s_{1}^{\prime}, s_{2}^{\prime}, \ldots, s_{2 l-2}^{\prime}\right\}$ (if $l \geqslant 2$ only) each be sets of distinct rows and $\left\{c_{1}, c_{2}, c_{3}, c_{2}^{\prime}, c_{3}^{\prime}, \ldots, c_{2 l+1}^{\prime}\right\}$ be a set of distinct columns. Let $R$ be the set of cells:

$$
\left\{\left(r_{1}, c_{1}\right),\left(r_{1}, c_{2}\right),\left(r_{2}, c_{1}\right),\left(r_{2}, c_{3}\right),\left(r_{3}, c_{2}\right),\left(r_{3}, c_{3}\right)\right\} .
$$


Defining $c_{1}^{\prime}=c_{1}$, let $R^{\prime}$ be the set of cells:

$$
\left\{\left(r_{1}^{\prime}, c_{1}^{\prime}\right),\left(r_{2 l+1}^{\prime}, c_{2 l+1}^{\prime}\right)\right\} \cup\left\{\left(r_{i}^{\prime}, c_{i+1}^{\prime}\right),\left(r_{i+1}^{\prime}, c_{i}^{\prime}\right) \mid 1 \leqslant i \leqslant 2 l\right\} .
$$

Let $U_{1}$ be the set of cells:

$$
\left\{\left(u_{1}, c_{1}\right),\left(u_{1}, c_{2}\right),\left(u_{1}, c_{2}^{\prime}\right),\left(u_{1}, c_{3}^{\prime}\right),\left(u_{2}, c_{1}\right),\left(u_{2}, c_{2}\right),\left(u_{2}, c_{2}^{\prime}\right),\left(u_{2}, c_{3}^{\prime}\right)\right\} .
$$

Let $U_{2}$ be the set of cells:

$$
\left\{\left(u_{3}, c_{1}\right),\left(u_{3}, c_{2}\right),\left(u_{3}, c_{3}\right),\left(u_{3}, c_{2}^{\prime}\right),\left(u_{4}, c_{1}\right),\left(u_{4}, c_{2}\right),\left(u_{4}, c_{3}\right),\left(u_{4}, c_{2}^{\prime}\right)\right\} .
$$

For each $i, 1 \leqslant i \leqslant l-1$, let $S_{i}^{\prime}$ be the set of cells

$$
S_{i}^{\prime}=\left\{\left(s_{2 i-1}^{\prime}, c_{j}^{\prime}\right),\left(s_{2 i}^{\prime}, c_{j}^{\prime}\right) \mid 2 i \leqslant j \leqslant 2 i+3\right\} .
$$

Suppose furthermore that each of the above sets of cells are pairwise disjoint. Then the edges corresponding to the union of these sets of cells decompose into 4-cycles.

Proof. The following sets of cells each correspond to 4-cycles:

$$
\begin{array}{lll}
\left\{\left(r_{1}, c_{1}\right),\left(r_{1}, c_{2}\right),\left(u_{1}, c_{1}\right),\left(u_{1}, c_{2}\right)\right\}, & \left\{\left(r_{2}, c_{1}\right),\left(r_{2}, c_{3}\right),\left(u_{3}, c_{1}\right),\left(u_{3}, c_{3}\right)\right\}, \\
\left\{\left(r_{3}, c_{2}\right),\left(r_{3}, c_{3}\right),\left(u_{4}, c_{2}\right),\left(u_{4}, c_{3}\right)\right\}, & \left\{\left(r_{1}^{\prime}, c_{1}\right),\left(r_{1}^{\prime}, c_{2}^{\prime}\right),\left(u_{4}, c_{1}\right),\left(u_{4}, c_{2}^{\prime}\right)\right\}, \\
\left\{\left(r_{2}^{\prime}, c_{1}\right),\left(r_{2}^{\prime}, c_{3}^{\prime}\right),\left(u_{2}, c_{1}\right),\left(u_{2}, c_{3}^{\prime}\right)\right\}, & \left\{\left(u_{2}, c_{2}\right),\left(u_{2}, c_{2}^{\prime}\right),\left(u_{3}, c_{2}\right),\left(u_{3}, c_{2}^{\prime}\right)\right\} .
\end{array}
$$

\begin{tabular}{|c|c|c|c|c|c|}
\hline & $c_{1}$ & $c_{2}$ & $c_{3}$ & $c_{2}^{\prime}$ & \\
\hline$r_{1}$ & 1 & 1 & & & \\
\hline$r_{2}$ & 2 & & 2 & & \\
\hline$r_{3}$ & & 3 & 3 & & \\
\hline$r_{1}^{\prime}$ & 4 & & & 4 & \\
\hline$r_{2}^{\prime}$ & 5 & & & & 5 \\
\hline$r_{3}^{\prime}$ & & & & 6 & 6 \\
\hline$u_{1}$ & 1 & 1 & & 6 & 6 \\
\hline$u_{2}$ & 5 & 7 & & 7 & 5 \\
\hline$u_{3}$ & 2 & 7 & 2 & 7 & \\
\hline$u_{4}$ & 4 & 3 & 3 & 4 & \\
\hline
\end{tabular}

\begin{tabular}{|c|c|c|c|c|c|c|c|}
\hline & $c_{1}$ & $c_{2}$ & $c_{3}$ & $c_{2}^{\prime}$ & $c_{3}^{\prime}$ & $c_{4}^{\prime}$ & $c_{5}^{\prime}$ \\
\hline$r_{1}$ & 1 & 1 & & & & & \\
\hline$r_{2}$ & 2 & & 2 & & & & \\
\hline$r_{3}$ & & 3 & 3 & & & & \\
\hline$r_{1}^{\prime}$ & 4 & & & 4 & & & \\
\hline$r_{2}^{\prime}$ & 5 & & & & 5 & & \\
\hline$r_{3}^{\prime}$ & & & & 6 & & 6 & \\
\hline$r_{4}^{\prime}$ & & & & & 7 & & 7 \\
\hline$r_{5}^{\prime}$ & & & & & & 8 & 8 \\
\hline$u_{1}$ & 1 & 1 & & 10 & 10 & & \\
\hline$u_{2}$ & 5 & 9 & & 9 & 5 & & \\
\hline$u_{3}$ & 2 & 9 & 2 & 9 & & & \\
\hline$u_{4}$ & 4 & 3 & 3 & 4 & & & \\
\hline$s_{1}^{\prime}$ & & & & 10 & 10 & 8 & 8 \\
\hline$s_{2}^{\prime}$ & & & & 6 & 7 & 6 & 7 \\
\hline
\end{tabular}

The remaining 4-cycles in the decomposition may be found in the same manner as in Lemma 4.

Example 12. We exhibit the cases $l=1$ and $l=2$ from the previous lemma in the arrays below.

The preceding lemmas combine to apply the following. 
Corollary 13. Let $C_{1}$ and $C_{2}$ be two cell-disjoint cycles, each of length congruent to 2 (mod 4) within a decomposition array $M$ (which satisfies (P1) and (P2)). Suppose furthermore that $C_{1}$ and $C_{2}$ share at most one column, and that the cells of $C_{1}$ and $C_{2}$ are all unavailable. Suppose that:

- for each subset of 6 columns, there exist at least 1 pair from $\mathcal{P}_{R}$ which is available in all of these columns (condition (Q1)); and

- for each subset of 4 columns, there exist at least 4 elements of $\mathcal{P}_{R}$ which are available in all of these columns (condition (Q2)).

Then, there exists a trade $\mathcal{D}_{1}$ of $C_{1}, C_{2}$ and 4-cycles whose edges correspond to available cells with a set $\mathcal{D}_{2}$ of 4-cycles only. Moreover, after reclassifying any available cells from $\mathcal{D}_{1}$ as unavailable, the matrix $M$ still satisfies properties (P1) and (P2).

Proof. The idea is similar to Corollary 6. Suppose first that $C_{1}$ is a 6-cycle sharing a column with $C_{2}$. Applying Lemma 11, we choose pairs from $\mathcal{P}_{R}$ in the order:

$$
\left\{u_{1}, u_{2}\right\},\left\{u_{3}, u_{4}\right\},\left\{s_{1}^{\prime}, s_{2}^{\prime}\right\},\left\{s_{3}^{\prime}, s_{4}^{\prime}\right\}, \ldots,\left\{s_{2 l-3}^{\prime}, s_{2 l-2}^{\prime}\right\} .
$$

Condition (Q2) allows us to make these choices so that the sets $R^{\prime}, U_{1}, U_{2}$ and $S_{i}^{\prime}(1 \leqslant$ $i \leqslant l-1)$ are each pairwise disjoint.

Next, suppose that $C_{1}$ and $C_{2}$ are disjoint. We seek to apply Lemma 9 in this case. Condition (Q1) allows us to choose the pair $\left\{u_{1}, u_{2}\right\}$ so that $U_{1}$ is disjoint from $C_{1}$ and $C_{2}$. Condition (Q2) allows us to choose the pair $\left\{u_{3}, u_{4}\right\}$ so that $U_{2}$ is disjoint from $C_{1}, C_{2}$ and $U_{1}$. Since $C_{1}$ and $C_{2}$ are disjoint, condition (Q2) allows us to choose the remaining pairs from $\mathcal{P}_{R}$ so that the required sets are disjoint, in the order:

$$
\left\{s_{1}, s_{2}\right\},\left\{s_{3}, s_{4}\right\}, \ldots,\left\{s_{2 k-3}, s_{2 k-2}\right\},\left\{s_{1}^{\prime}, s_{2}^{\prime}\right\},\left\{s_{3}^{\prime}, s_{4}^{\prime}\right\}, \ldots,\left\{s_{2 l-3}^{\prime}, s_{2 l-2}^{\prime}\right\} .
$$

Finally, suppose that $C_{1}$ and $C_{2}$ each have length at least 10 and share one column. Again we apply Lemma 9. We proceed as above, taking notice of the extra constraint $c_{2 l+1}^{\prime}=c_{2 k+1}$. This potentially effects our choice for the final pair; we must ensure that $S_{l-1}^{\prime}$ and $S_{k-1}$ are disjoint. Since (Q2) allows four choices of pairs, this is no problem. (We only possibly need four choices in the case where $C_{1}$ and $C_{2}$ are 10-cycles sharing a common vertex.)

\section{The main result}

In this section we prove the main result of the paper (Theorem 3).

Let $X=\left\{x_{1}, x_{2}, \ldots, x_{m}\right\}$ and $Y=\left\{y_{1}, y_{l}, \ldots, y_{n}\right\}$. As outlined in Section 2, we associate with $G$ an $m \times n$ matrix $M$ where cell $(i, j)$ corresponds with the edge $\left\{x_{i}, y_{j}\right\}$. Our goal is to decompose $G$ into 4-cycles. We achieve this by first packing the empty cells of $G$ with 4 -cycles in a structured way (creating a set of 4 -cycles $\mathcal{C}_{0}$ ), then in a greedy way (creating a set of 4 -cycles $\mathcal{C}_{1}$ ). The remaining edges must decompose into even length 
cycles (the set $\mathcal{C}_{2}$ ); using the corollaries above, we trade these with cycles from $\mathcal{C}_{0}$ to obtain the full decomposition.

Cells from $M$ corresponding to edges not in $G$ are filled with the symbol 0 (these obviously will not be used in any 4-cycle.) As previously, let $\mathcal{P}_{R}$ be some partition of the rows into pairs (and at most one singleton) and let $\mathcal{P}_{C}$ be a partition of the columns into pairs. For each $P_{1}$ and $P_{2}$ such that $\left|P_{1}\right|=2, P_{1} \in \mathcal{P}_{R}$ and $P_{2} \in \mathcal{P}_{C}$, if cell $(i, j)$ is empty for each $i \in P_{1}$ and $j \in P_{2}$, then we place a 4-cycle on these four cells. Let the set of these 4 -cycles be $\mathcal{C}_{0}$. (Some of the 4-cycles from $\mathcal{C}_{0}$ will be adjusted later in our proof.) We initially classify every cell from $\mathcal{C}_{0}$ as available and the remaining cells in $M$ as unavailable. Note that $M$ initially satisfies conditions (P1) and (P2) (see Section 2).

In each column of $M$, at most $(1-d) m-1$ cells contain 0 . Let $P$ be a pair of columns from $\mathcal{P}_{C}$; at most $2(1-d) m-2$ cells within columns from $P$ contain 0 . Thus each column has at most $2(1-d) m-2$ pairs of cells which are classified unavailable.

Next, pack as many 4-cycles as possible in the remaining empty cells. Let the set of such 4 -cycles be $\mathcal{C}_{1}$, where $\mathcal{C}_{1}$ is disjoint from $\mathcal{C}_{0}$. (These 4 -cycles remain unchanged for the rest of the proof.)

After the edges from the 4-cycles of $\mathcal{C}_{0} \cup \mathcal{C}_{1}$ are removed from $G$, the resultant graph $G^{\prime}$ is even; thus $G^{\prime}$ decomposes into a set of cycles $\mathcal{C}_{2}$. Since $\mathcal{C}_{0} \cup \mathcal{C}_{1}$ is a maximal packing of 4-cycles, each cycle from $\mathcal{C}_{2}$ contains at least 6 edges. Now, as each vertex of $G$ has even degree and $Y$ has even size, the number of edges in $G$ is divisible by 4 . It follows that the total number of edges in $\mathcal{C}_{2}$ is divisible by 4 . Thus there is a partition $\mathcal{Q}$ of $\mathcal{C}_{2}$ into singletons or pairs, so that the total number of edges in each element of $\mathcal{Q}$ is divisible by 4 . That is, each element of $\mathcal{Q}$ contains either a single cycle with length divisible by 4 or two cycles, each of which have lengths congruent to 2 (mod 4). From Corollary 8, we may also assume in the latter case that the two cycles share at most one common column.

By Corollaries 6 and 13, we can take each element of $\mathcal{Q}$ and identify extra available cells, then trade the corresponding edges with 4-cycles, provided there are enough available choices for available cells. After each trade, any available cells used are reclassified as unavailable. Observe that conditions $(\mathrm{P} 1)$ and $(\mathrm{P} 2)$ are invariant to the application of trades.

The trades are in effect using some edges from some of the cycles from $\mathcal{C}_{0}$. However, after all trades have been applied, within each pair of rows $P \in \mathcal{P}_{R}$, (P2) implies that we have used each edge from $\mathcal{C}_{0}$ from an even number of columns. Thus any remaining available cells from $P$ may be decomposed into 4-cycles.

Thus it remains to ensure that there are always enough available cells to perform the required trades. Consider a column $c$. From above, at most $2 m(1-d)-2$ pairs of rows from $\mathcal{P}_{R}$ are unavailable. Thus at most $4 m(1-d)-3$ cells in column $c$ are involved with cycles from $C_{2}$. (The extra 1 here is a possible contribution from the final row, when $m$ is odd.) It then follows that at most $2 m(1-d)-2$ cycles from $C_{2}$ use column $c$, since each cycle uses either 2 or 0 cells from a column.

So, if we are dealing with a particular cycle $C \in \mathcal{C}_{2}$ which intersects a column $c$, we have already dealt with at most $2 m(1-d)-3$ other cycles from $\mathcal{C}_{2}$ which also involve column $c$, each of which may have "used" up to 3 pairs of rows from $\mathcal{P}_{R}$ containing cells 
from $\mathcal{C}_{0}$. (The maximum number may occur via an application of Lemma 9 or Lemma 11.) Thus for a given column $c$, at most $2 m(1-d)-2+3(2 m(1-d)-3)=8 m(1-d)-11$ pairs of rows from $\mathcal{P}_{R}$ are either unavailable or used by another cycle from $\mathcal{C}_{2}$.

It follows that amongst any 6 columns of $C$, there exist at least $(m-1) / 2-6(8 m(1-d)-$ $11)=m(96 d-95) / 2+131 / 2>1$ pair of rows from $\mathcal{P}_{R}$ which is simultaneously available for each of these 6 columns, and moreover have not been previously used. Similarly, amongst any 4 columns of $C$ there exist at least at least $(m-1) / 2-4(8 m(1-d)-11)=$ $m(64 d-63) / 2+87 / 2>4$ pairs of rows from $\mathcal{P}_{R}$ which are simultaneously available for each of these 4 columns, and moreover have not been previously used.

By Corollaries 6 and 13 and the above, we may thus perform the necessary trades so that $\mathcal{C}_{0} \cup \mathcal{C}_{1}$ (and thus $G$ ) decomposes into 4-cycles. This completes the proof of Theorem 3 .

\section{Lower bounds on the minimum degree}

Using notation similar to [13], we define $b(n)$ to be the least integer such that whenever a bipartite graph $G$ with partite sets of size $n$ satisfies $(\mathrm{C} 1)$ and $(\mathrm{C} 2)$ and has minimum degree at least $b(n)$, then $G$ decomposes into 4-cycles. We furthermore let $B=\lim \sup _{n \rightarrow \infty} b(n) / n$. and $B_{\text {even }}=\limsup _{n \rightarrow \infty} b(2 n) /(2 n)$. Thus our main result Theorem 3 implies that $B_{\text {even }} \leqslant 95 / 96$. The following theorem suggests that $B \geqslant 1 / 2$; we conjecture that $B=B_{\text {even }}=1 / 2$.

Theorem 14. For each even $n, n \geqslant 6$, there exists an even bipartite graph with partite sets of size $n$, size divisible by 4 and minimum degree $4\lfloor(n+2) / 8\rfloor-2$ which does not decompose into 4-cycles.

Proof. Let $X=X_{1} \cup X_{2}, Y=Y_{1} \cup Y_{2}$ be the partite sets of $G$, where $X_{1} \cap X_{2}=Y_{1} \cap Y_{2}=\emptyset$. Let $\left|X_{1}\right|=\left|Y_{1}\right|=4\lfloor(n+2) / 8\rfloor-1$. Remove from $G$ all edges between $X_{1}$ and $Y_{2}$, all edges between $X_{2}$ and $Y_{1}$, a 1-factor between $X_{1}$ and $Y_{1}$ and a 1-factor between $X_{2}$ and $Y_{2}$. Observe that $G$ is an even graph with minimum degree $4\lfloor(n+2) / 8\rfloor-2$ and consists of two components, each with size (number of edges) congruent to 2 (modulo 4 ).

\section{Conclusion}

There are several ways in which the lemmas of the previous section may be strengthened. As far as I can see the changes do not improve the main result. However we mention them here in case they are applicable to future results.

Firstly, in Lemma 4, the lemma still holds even if $S_{i}$ and $S_{i+1}$ share the same row set (for any $i$ such that $1 \leqslant i \leqslant k-2$ ); it is not hard to see how the decomposition may be modified to cope with this scenario. It follows in Corollary 6 , we only require one valid choice for the pair of rows $P$ (rather than 2). Similarly, in Lemma 9 we may have $S_{1}$ or $S_{1}^{\prime}$ sharing the same pair of rows as $U_{2}$, or $S_{i}$ and $S_{i+1}$ sharing the same row set (for any $i$ such that $1 \leqslant i \leqslant k-2$ ) or $S_{i}^{\prime}$ and $S_{i+1}^{\prime}$ sharing the same row set (for any $i$ such that 
$1 \leqslant i \leqslant l-2)$. In Lemma 11, we may have $S_{1}^{\prime}$ sharing the same pair of rows as $U_{1}$, or $S_{i}^{\prime}$ and $S_{i+1}^{\prime}$ sharing the same row set (for any $i$ such that $1 \leqslant i \leqslant l-2$ ). Consequently, in Condition (Q2) of Corollary 13, 2 elements of $\mathcal{P}$ suffice.

Furthermore, it might be possible to ensure that for any column, there exists at most one (or some finite number) of trades which use available cells from three distinct pairs of rows. This would allow us to improve our bound from 95/96 to 71/72. I have decided that attempting such an approach risks making the proof convoluted. It seems unlikely that $71 / 72$ is the best possible bound, so it may not be worth the effort.

We have chosen arbitrary pairings $\mathcal{P}_{R}$ of the rows and $\mathcal{P}_{C}$ columns. It may be possible to choose these in some wise fashion, significantly increasing the number of cycles in $\mathcal{C}_{0}$. However, techniques above are unlikely to prove that $B=1 / 2$ (see the previous section); to do so would require innovation not directly suggested in this paper.

Finally, there is no obvious reason why the main result in this paper should not be true when $n$ is odd. This remains an open problem.

\section{References}

[1] P. Balister, Packing closed trails into dense graphs, Journal Combin. Theory Ser. B 88 (2003), 107-118.

[2] B. Barber, D. Kühn, A. Lo and D. Osthus, Edge-decompositions of graphs with high minimum degree, Preprint, 2014. arXiv:1410.5750

[3] D. Bryant and N. Cavenagh, Decomposing graphs with high minimum degree into 4-cycles, J. Graph Theory (to appear). doi:10.1002/jgt.21816.

[4] D. Bryant and S. El-Zanati, Graph Decompositions, in: C.J. Colbourn and J.H. Dinitz (ed.), Handbook of Combinatorial Designs, 2nd Edition (Chapman and Hall/CRC, Boca Raton, 2007).

[5] Y. Caro and R. Yuster, List decomposition of graphs, Discrete Math. 253 (2002), $67-77$.

[6] N. Cavenagh and E. Billington, Decomposition of complete multipartite graphs into cycles of even length, Graphs Combin. 16 (2000), 49-65.

[7] D. Dor and M. Tarsi, Graph decomposition is NP-complete: A complete proof of Hoyler's conjecture, SIAM J. Comput. 26 (1997), 1166-1187.

[8] T. Gustavsson, Decompositions of large graphs and digraphs with high minimum degree, Ph.D Thesis, University of Stockholm, 1991.

[9] C.C. Lindner and A. Rosa, Mongoamous decompositions of complete bipartite graphs, symmetric H-squares, and self-orthogonal 1-factorizations, Australas. J. Combin. 20 (1999), 251-256.

[10] C. St. J. A. Nash-Williams, An unsolved problem concerning decomposition of graphs into triangles, in: Combinatorial theory and its applications III ed., pp. 1179-1183, 1970. 
[11] D. Sotteau, Decomposition of $K_{m, n}\left(K_{m, n}^{*}\right)$ into cycles (circuits) of length $2 k$, J. Combin. Theory Ser. B. 30 (1981).

[12] R.M. Wilson, Decompositions of complete graphs into subgraphs isomorphic to a given graph, Congr. Numer. 15 (1976), 647-659.

[13] R. Yuster, The decomposition threshold for bipartite graphs with minimum degree one, Random Struct. Alg. 21 (2002), 121-134. 